\title{
Receptor for advanced glycation end products gene polymorphisms in cardiac syndrome $X$
}

\author{
BURAK ÖNAL ${ }^{1}$, DENIZ ÖZEN ${ }^{2}$, BÜLENT DEMIR ${ }^{3}$, AHMET G. AKKAN $^{2}$ and SIBEL ÖZYAZGAN ${ }^{2}$ \\ ${ }^{1}$ Department of Medical Pharmacology, Faculty of Medicine, Biruni University, Istanbul 34010; \\ ${ }^{2}$ Department of Medical Pharmacology, Cerrahpaşa Medical Faculty, Istanbul University-Cerrahpaşa, Istanbul 34096; \\ ${ }^{3}$ Department of Cardiology, Bakirkoy Dr. Sadi Konuk Training and Research Hospital, \\ University of Health Sciences, Istanbul 34147, Turkey
}

Received April 18, 2019; Accepted July 5, 2019

DOI: $10.3892 /$ br.2019.1231

\begin{abstract}
Endothelial and microvascular dysfunction serve important roles in the formation and pathogenesis of cardiac syndrome X (CSX). Expression of receptor for advanced glycation end products (RAGE) is suggested to be increased in several conditions, including diabetes, inflammation and vascular diseases. In the present study, RAGE gene polymorphisms in patients with CSX and healthy controls were investigated. A total of 114 patients, diagnosed with CSX using coronary angiography results following complaints of angina and objective ischemia, and 103 healthy controls participated in the study. Whether there was a difference in genotype distributions of RAGE gene -374T/A, -429T/C and Glys82Ser polymorphisms between patients with CSX and healthy controls was investigated. Following DNA isolation from blood samples of the participants, the polymorphic regions were examined by quantitative polymerase chain reaction, and the genotyping results were statistically analyzed. When the genotypic distributions of $-374 \mathrm{~T} / \mathrm{A},-429 \mathrm{~T} / \mathrm{C}$ and Gly82Ser polymorphisms were investigated in patients with CSX and healthy controls, no statistically significant differences were identified between the two groups $(\mathrm{P}>0.05)$. Likewise, no statistically significant differences were observed in the allelic distributions of all 3 polymorphic regions $(\mathrm{P}>0.05)$. To the best of our knowledge, the present study also investigated the association between CSX and RAGE gene polymorphisms for the first time. No statistically significant differences in $R A G E$ gene polymorphisms between the CSX and control groups were observed. We hypothesized that significant results may
\end{abstract}

Correspondence to: Professor Sibel Özyazgan, Department of Medical Pharmacology, Cerrahpaşa Medical Faculty, Istanbul University-Cerrahpaşa, Cerrahpaşa Mahallesi, 53 Koca Mustafapaşa Cad., Istanbul 34096, Turkey

E-mail: ozyazgans@yahoo.com

Key words: cardiac syndrome $\mathrm{X}$, inflammation, microvascular dysfunction, polymorphism, receptor for advanced glycation end products be obtained by increasing the numbers of patients and healthy controls in future studies.

\section{Introduction}

Cardiac syndrome $\mathrm{X}$ (CSX) is defined as an angina-like pain despite positive treadmill exercise tests and normal coronary arteriograms (1-3). The pathogenesis of CSX is not completely understood and diagnosis of the syndrome is based on eliminating other potential causes of chest pain (4). Previous studies have suggested that coronary microvascular dysfunction may be one of the primary underlying causes of ischemic heart disease, and of chest pain without any significant coronary obstruction (5). In addition, myocardial ischemia, which is a result of microvascular and endothelial dysfunctions, is also considered to be one of the causative factors of angina (6). Coronary microvascular dysfunction is suggested to be the primary reason for the visible symptoms in a number of patients experiencing angina attacks without a significant cardiac or systemic disease (7).

Inflammation is known to be involved in endothelial and vascular dysfunction. Additionally, previous studies have suggested that inflammation may serve a significant role in the pathogenesis of CSX $(8,9)$. The role of microvascular dysfunction in CSX has been studied by examining the response to vasoactive stimuli that occurs in coronary flow (1), and it is considered to be the primary pathological cause in numerous patients (10).

Advanced glycation end products (AGE) are spontaneously modified structures or newly formed compounds of proteins, lipids or nucleic acids with non-enzymatic glycation and glycosylation in oxidation accelerating environments (11). The receptor for AGE (RAGE), which is a member of the immunoglobulin superfamily and a transmembrane multiligand receptor, is primarily active in the response to acute and chronic stress $(12,13)$. Upregulation of RAGE is observed in diabetes, vascular, inflammatory and neurodegenerative diseases, and it is hypothesized to serve a role in the pathogenesis and complications of these diseases $(14,15)$. RAGE is generally expressed in vascular endothelial and epithelial cells, smooth muscles, macrophages and fibroblasts (16). Under normal physiological conditions, RAGE is usually expressed in small 
amounts in adult tissues, while its expression is upregulated in pathological vascular conditions. The receptor is suggested to serve an important role in the progression of coronary artery disease (17). In addition, previous studies have suggested that different $R A G E$ gene polymorphisms are associated with a number of diseases including coronary artery disease, heart failure, nephropathy, chronic renal failure and diabetes (18).

To the best of our knowledge, no experiments have been conducted to investigate the association between CSX disease and RAGE gene polymorphisms. Therefore, the present study compared patients with CSX and control subjects from the Turkish population in the terms of 429T/C, -374T/A and G82S single nucleotide polymorphisms (SNPs), which have been previously evaluated in a number of studies due to their associations with a number of cardiovascular diseases (19-21).

\section{Materials and methods}

Patient selection. The present study was approved by Ethical Committee of Cerrahpaşa Medical Faculty (approval no. 2015/279734) and was performed in accordance with Helsinki Declaration of World Health Organization. Only patients and controls, who agreed to give written informed consent, were included.

The study was designed as a cohort study, and was conducted from September 2015 to January 2016; a total of 217 subjects were included. Patients were admitted to the Cardiology Clinic of Bakırköy Dr. Sadi Konuk Training and Research Hospital (Istanbul, Turkey) with complaints of angina and objective ischemia. If they were diagnosed with CSX according to results of coronary angiography, they were included in the study cohort ( $\mathrm{n}=114,76$ females and 38 males; average age, $52.0 \pm 11.9$ years). The control group was formed of 103 consecutive subjects with similar demographic data but without CSX diagnoses.

Exclusion criteria were as follows: Previous diagnosis of coronary artery disease, valvular heart disease, congestive heart failure, cardiomyopathy, pericarditis or myocarditis; abnormality in liver enzyme tests; creatinine $>2 \mathrm{mg} / \mathrm{dl}$; presence of active infection; and diagnosis of pulmonary thromboembolism or acute coronary syndrome.

The diagnosis of CSX was performed according to 2006 Stable Angina Guidelines of European Society of Cardiology (22). As suggested in the guidelines, patients were diagnosed with CSX if they met the following criteria: Angina being induced by typical exercise; positive ECG in exercise stress or evidence of ischemia by other screening method; and normal epicardial coronary arteries in angiography.

The control group was recruited from the subjects admitted to the cardiology clinic with angina pain, but with no ischemic change following an exercise test and a risk of cardiovascular events $<10 \%$, according to Framingham risk score (23).

Detection of objective ischemia. All the patients underwent an exercise test, which was modified according to Bruce's protocol (24). Based on the results of ECG exercise records, after 60-80 milliseconds from $\mathrm{J}$ point, at least 2 derivations and at least $1 \mathrm{~mm}$ horizontal or downsloping ST segment depression of ischemic change were considered as abnormal. Patients exhibiting evidence of objective ischemia in the exercise test underwent coronary angiographies using the Judkins technique during right femoral artery bypass.

Genotyping. Blood samples were collected in K3-EDTA tubes. Genomic DNA isolation was performed using peripheral blood from $200 \mu \mathrm{l}$ blood samples, according to the manufacturer's protocol of the Invitrogen PureLink ${ }^{\circledR}$ Genomic DNA Mini kit (cat. no. K1820-02; Invitrogen; Thermo Fisher Scientific, Inc.). Absorbance $\left(\mathrm{A}_{260} / \mathrm{A}_{280}\right)$ values of the extracted DNA samples were calculated using the NanoDrop 2000 system, and the minimum acceptable concentration level was determined as $1.8 \pm 0.2$. The DNA samples were stored in $-20^{\circ} \mathrm{C}$ until further use.

RAGE gene -374T>A (rs1800624); -429T>C (rs1800625) and Gly82Ser (rs2070600) polymorphisms were detected by quantitative polymerase chain reaction using the Applied Biosystems 7500 Fast system (Applied Biosystems; Thermo Fisher Scientific, Inc.) and gene fragment-specific TaqMan probes (Thermo Fisher Scientific, Inc.; cat. no. 4351379), according to a VIC- and FAM-labeled allelic discrimination method for each polymorphism: rs1800624 (assay ID., C_3293837_1_); rs1800625 (assay ID., C_8848033_1_); and rs2070600 (assay ID., C_15867521_20). The primers were as follows: rs1800624, forward, 5'-CCCATCTTGATTGCGCAAAGTT-3' and reverse, 5'-TCAAAAAACATGAGAAACCCCAGAA-3' with the reference gene, 5'-CCAGACTGTTGTCTGCAAGGGTGCA[A/T] TTGGGCCTGCATCATGAAGGCAAGG-3'; rs1800625, forward, 5'-CCCATCTTGATTGCGCAAAGTT-3' and reverse, 5'-TCAAAAAACATGAGAAACCCCAGAA-3' with the reference gene, 5'-GGAACAGGAGAGAAACCTGTTTGGA[A/G] CTTCGTGAA AGA AAATCATTTTTT T-3'; rs2070600, forward, 5'-TGGTCTCCTTTCCATTCCTGTTCA-3' and reverse, 5'-CCGTCACTCTGCCTCACAGTCC-3' with the reference gene, 5'-CCGACAGCCGGAAGGAAGAGGGAGC[C/T] GTTGGGAAGGACACGAGCCACACTG-3'. The qPCR conditions were denaturation for $10 \mathrm{~min}$ at $95^{\circ} \mathrm{C}$, followed by 35 cycles of denaturation for $15 \mathrm{sec}$ at $95^{\circ} \mathrm{C}$, annealing and extension for $1 \mathrm{~min}$ at $60^{\circ} \mathrm{C}$, and a final extension for $1 \mathrm{~min}$ at $60^{\circ} \mathrm{C}$. The $2^{-\Delta \Delta \mathrm{Cq}}$ method was used to quantify the results (25).

Statistical analysis. Data were analyzed with the SPSS software v22.0 for Windows (IBM Corp.). Descriptive data are presented as the mean \pm standard deviation, and median (range), frequency and ratio. The distribution of the variables was analyzed by Kolmogorov-Smirnov test. Qualitative data were analyzed Mann-Whitney U and independent Student's t-tests. Quantitative data were measured using the $\chi^{2}$ test. $\mathrm{P}<0.05$ was considered to indicate a statistically significant difference.

\section{Results}

Demographic patient data. The comparisons between demographic, clinical and laboratory data of the patients are summarized in Tables I and II. In Table I, demographic characteristics of the CSX and control groups were compared based on age, sex, BMI and smoking habits; no significant 
Table I. Patient demographic data.

\begin{tabular}{|c|c|c|c|c|c|c|c|}
\hline \multirow[b]{2}{*}{ Categories } & \multicolumn{3}{|c|}{ Patients $(n=114)$} & \multicolumn{3}{|c|}{ Controls $(n=103)$} & \multirow[b]{2}{*}{ P-value } \\
\hline & Mean \pm SD & Median (range) & $\mathrm{n}(\%)$ & Mean \pm SD & Median (range) & n $(\%)$ & \\
\hline Age & $52.0 \pm 11.9$ & $52(17-75)$ & & $50.3 \pm 10.5$ & $51(22-73)$ & & 0.123 \\
\hline BMI & $24.8 \pm 3.0$ & $24(19-32)$ & & $25.0 \pm 2.2$ & $25(20-30)$ & & 0.335 \\
\hline \multicolumn{8}{|l|}{ Sex } \\
\hline Female & & & $76(67)$ & & - & $61(59)$ & 0.256 \\
\hline Male & & & $38(33)$ & & - & $42(41)$ & \\
\hline Smoking & & & $36(32)$ & & - & $30(29)$ & 0.695 \\
\hline T2DM & & & $27(24)$ & & - & $8(8)$ & 0.001 \\
\hline Family history & & & $47(41)$ & & - & $17(17)$ & $<0.001$ \\
\hline $\mathrm{HT}$ & & & $31(27)$ & & - & $13(13)$ & 0.008 \\
\hline
\end{tabular}

Age and BMI data were analyzed using Mann-Whitney U test, all other data using a $\chi^{2}$ test. BMI, body mass index; T2DM, type 2 diabetes mellitus; HT, hypertension; SD, standard deviation.

Table II. Clinical laboratory results.

\begin{tabular}{|c|c|c|c|c|c|}
\hline \multirow[b]{2}{*}{ Serum markers } & \multicolumn{2}{|c|}{ Patients } & \multicolumn{2}{|c|}{ Controls } & \multirow[b]{2}{*}{ P-value } \\
\hline & Mean \pm SD & Median (Min-Max) & Mean \pm SD & Median (Min-Max) & \\
\hline FPG & $105.5 \pm 30.3$ & $98(74-266)$ & $93.3 \pm 17.4$ & $89(71-196)$ & $<0.001$ \\
\hline ALT & $20.8 \pm 15.1$ & $18(7-152)$ & $23.6 \pm 14.9$ & $20(8-91)$ & 0.075 \\
\hline AST & $21.1 \pm 7.9$ & $20(6-74)$ & $21.9 \pm 10.7$ & $19(11-103)$ & 0.968 \\
\hline GGT & $25.1 \pm 17.2$ & $21(9-156)$ & $26.3 \pm 13.2$ & $24(7-74)$ & 0.088 \\
\hline HDL-C & $47.7 \pm 11.6$ & $47(24-79)$ & $49.3 \pm 17.1$ & $46(30-179)$ & 0.817 \\
\hline LDL-C & $123.5 \pm 40.2$ & $121(46-254)$ & $122.7 \pm 32.6$ & $118(44-224)$ & 0.962 \\
\hline TG & $142.9 \pm 83.9$ & $123(41-603)$ & $131.5 \pm 71.6$ & $113(17-452)$ & 0.373 \\
\hline Total cholesterol & $199.5 \pm 44.5$ & $199(101-343)$ & $202.2 \pm 38.4$ & $205(114-318)$ & 0.718 \\
\hline Uric acid & $5.0 \pm 2.6$ & $5(2-28)$ & $4.9 \pm 1.4$ & $5(1-8)$ & 0.631 \\
\hline
\end{tabular}

Total cholesterol data were compared using an independent Student's t-test, all other data were analyzed using the Mann-Whitney U test. ALT, alanine aminotransferase; AST, aspartate aminotransferase; FPG, fasting plasma glucose; GGT, $\gamma$-glutamyl transferase; HDL-C, high density lipoprotein cholesterol; LDL-C, low density lipoprotein cholesterol; TG, triglyceride; SD, standard deviation.

differences were observed among the groups. In addition, when CSX and control groups were compared based on family history, the presence of type 2 diabetes mellitus, and the presence of hypertension, statistically significant differences were identified $(\mathrm{P}<0.05$; Table I).

No significant differences between CSX and control groups regarding clinical parameters including aspartate aminotransferase, alanine aminotransferase, $\gamma$-glutamyl transferase high density lipoprotein cholesterol, low density lipoprotein cholesterol, triglycerides, total cholesterol and uric acid were observed $(\mathrm{P}>0.05)$. Conversely, the fasting plasma glucose levels in the CSX group were significantly increased compared with the control group $(\mathrm{P}<0.05$; Table II).

Genotyping data. Genetic distributions of the subjects are summarized in Table III. No significant difference was observed when the CSX and control groups were compared on the basis of genotypic distributions of different $R A G E$ genes, including the genotypic distribution of rs1800624 and rs1800625 (P>0.05; Table III). Similarly, no significant differences were observed between the CSX and control groups in the distribution of rs2070600 genotype (P>0.05; Table III). In addition, when the allele distributions of the polymorphisms in patients were compared with the control group, no significant difference was identified (Table IV).

\section{Discussion}

The results of the present study indicated that $R A G E$ gene rs1800624, rs1800625 and rs2070600 polymorphisms were not statistically different between CSX and control groups. Mostly microvascular dysfunction has been suggested to serve a role in the pathogenesis of CSX. Inflammation is 
Table III. Genotyping results of $R A G E$ polymorphisms.

\begin{tabular}{lcccc}
\hline Polymorphisms & Allele & $\begin{array}{c}\text { Patients } \\
\mathrm{n}(\%)\end{array}$ & $\begin{array}{c}\text { Controls } \\
\mathrm{n}(\%)\end{array}$ & P-value \\
\hline RAGE rs1800624 & AA & $40(35)$ & $38(37)$ & 0.471 \\
& AT & $54(47)$ & $53(51)$ & \\
& TT & $20(18)$ & $12(12)$ & \\
RAGE rs1800625 & CC & $4(4)$ & $1(1)$ & 0.213 \\
& TC & $11(10)$ & $16(16)$ & \\
& TT & $99(87)$ & $86(83)$ & \\
$R A G E$ rs2070600 & GG & $108(95)$ & $95(92)$ & 0.453 \\
& GA & $5(4)$ & $6(6)$ & \\
& AA & $1(1)$ & $2(2)$ & \\
\hline
\end{tabular}

Data were analyzed using $\chi^{2}$ test. RAGE, receptor for advanced glycation end products; SD, standard deviation.

indicated to have an important role in the formation of both endothelial and microvascular dysfunctions in $\operatorname{CSX}(8,26)$. An increase in C-reactive protein (CRP) levels is a marker of inflammation in the presence of injury, infection or pain (27). Arroyo-Espliguero et al (28) indicated that significantly increased levels of CRP were detected in patients with CSX compared with controls. It has also been established that inflammation alters the bioavailability of nitric oxide and has an important role in the pathogenesis of vascular dysfunction (29).

Even though cardiovascular diseases are polygenic conditions, genetic mutations may be involved in familial cardiovascular risk factors (30). Therefore, family history should be considered in the diagnosis of CSX. Besides, dyslipidemia, hypertension and obesity are cardiovascular risk factors, which may also increase the risk of type 2 diabetes (31); type 2 diabetes mellitus was suggested as a contributing factor to the risk of vascular complications when patients with the condition were compared with non-diabetic individuals (32). Impaired endothelial function is observed also in patients with type 2 diabetes mellitus (33). These data supported the observations of the present study, which demonstrated that even though healthy individuals and patients exhibited similar demographic attributes, there were statistically significant differences between the two groups in terms of family history, hypertension and type 2 diabetes mellitus $(\mathrm{P}<0.05)$. In previous studies, when patients with CSX were compared with healthy individuals, it was revealed that the patients with CSX had an elevated inflammatory response $(8,9)$. Based on these data, we investigated the association between CSX and 3 different cytokines, including pro-inflammatory cytokines, IL-6 and TNF- $\alpha$, and an anti-inflammatory cytokine IL-10 in a previous study. The results indicated that patients with CSX exhibited increased serum IL- 6 and IL-10 levels, but decreased serum TNF- $\alpha$ levels compared with the control group. Conversely, no significant differences in $I L-6, I L-10$ and $T N F-\alpha$ gene polymorphisms were observed between patients with CSX and healthy controls (34).

In other respects, RAGE is suspected to initiate a proinflammatory process and to cause the development of endothelial dysfunction by increasing oxidative stress at a microvascular level. As a result, vasoreactivity may be impaired and lead to ischemia at a microvascular level in the coronary artery system. Furthermore, RAGE may cause fibrosis at microvascular levels and attribute to CSX pathogenesis by decreasing the ability of the small arteries to dilate in the myocardium, and by causing an increase in the levels of microvascular resistance. A previous study demonstrated an increase of microvascular resistance in patients with CSX (4).

Data from previous studies suggest that CSX has a complex mechanism $(4,8,9)$ and RAGE may be involved in the pathogenesis of the disease. To the best of our knowledge, there are no studies examining RAGE levels in patients with CSX, emphasizing the requirement for new studies to investigate the topic.

RAGE is known to interact with certain members of the S100/Calgranulin Family and high mobility group Box-1 proteins; accumulation of these ligands is common in inflammation, cancer or neurodegenerative disorders (35). Binding of such ligands to RAGE causes chemotaxis and oxidative stress, and an enhanced production of pro-inflammatory molecules by triggering the self-expression of RAGE (36). These RAGE-mediated signals are involved not only in diabetes, atherosclerosis or Alzheimer's disease, but also in cardiac ischemia and acute peritoneal inflammation (12). Therefore, oxidative and carbonyl stresses cause an inflammatory response by damaging a number of biological compounds including protein, lipids and nucleic acids (11). In addition, in the tissues that are exposed to oxidative stress, RAGE contributes to vascular inflammation and atherosclerotic changes by interacting with AGE (13). These interactions lead to numerous biological responses, including cell migration and proliferation, and the activation of proinflammatory and prothrombotic pathways (37). The upregulation of these pathways and RAGE-mediated oxidative stress generation results in tissue damage (15). Expression of both proinflammatory cytokines, and transcription factor NF-KB, which is activated as a result of ligand-receptor binding, also imply that the RAGE-AGE interaction affects the inflammatory response (38). A previous study reported the RAGE-AGE interaction in activation of cell-cell signaling and in gene expression, indicating that RAGE expression serves a significant role in the pathogenesis of vascular and heart diseases (39). SNPs of $-429 / \mathrm{C}$ and $-374 \mathrm{~T} / \mathrm{A}$; and SNP in exon 3 of the gene (Gly82Ser) are among the most frequently studied polymorphisms in the $R A G E$ gene (38). In addition, polymorphisms of $-429 \mathrm{~T} / \mathrm{C}$ and 374T/A that have been identified in the RAGE promoter gene are considered to have effects on RAGE mRNA and protein expression (40). A previous study suggests that the AA genotype of the -374T/A polymorphism exhibits a protective effect against coronary atherosclerosis (41). Conversely, the G82S polymorphism has been observed in the ligand-binding section, affecting the ligand binding affinity, which may cause a number of diseases associated with an increase in oxidative stress and inflammation through RAGE activation (37). In vitro studies have demonstrated that the presence of the $-429 \mathrm{C}$ allele increases the transcriptional activity of the gene and 
Table IV. Allelic distribution results of RAGE polymorphisms.

\begin{tabular}{|c|c|c|c|c|}
\hline Polymorphisms & Allele & Patients, n (\%) & Controls, n (\%) & P-value \\
\hline \multirow[t]{2}{*}{$R A G E$ rs 1800624} & A & $134(58.8)$ & $129(62.6)$ & \multirow[t]{2}{*}{0.978} \\
\hline & $\mathrm{T}$ & $94(41.2)$ & $77(37.4)$ & \\
\hline \multirow[t]{2}{*}{$R A G E$ rs 1800625} & $\mathrm{C}$ & $19(8.3)$ & $18(8.7)$ & \multirow[t]{2}{*}{0.982} \\
\hline & $\mathrm{T}$ & $209(91.7)$ & $188(91.3)$ & \\
\hline \multirow[t]{2}{*}{$R A G E$ rs 2070600} & G & $221(96.9)$ & $196(95.1)$ & \multirow[t]{2}{*}{0.478} \\
\hline & A & $7(3.1)$ & $10(4.9)$ & \\
\hline
\end{tabular}

Data were analyzed using $\chi^{2}$ test. RAGE, receptor for advanced glycation end products.

increases RAGE expression by 2 -fold. Consequently, in the pathogenesis of heart disease, polymorphic regions have been closely examined as potential risk factors (39).

A number of studies have focused on polymorphisms within the RAGE gene, examining the effects on diabetic complications and the inflammatory response in atherosclerosis (15). In a cerebral cortex ischemia mouse model, RAGE was expressed in both macrophages and neurons, which led to neuronal cell death and inflammation (12). In addition, a previous study has indicated that RAGE may be involved in the initiation and progress of atherosclerosis (41). Bucciarelli et al (14) demonstrated an association between RAGE and cardiac dysfunction, through the observation of ischemic myocardial damage in $R A G E$-null mice.

In the study by Falcone et al (41), the -374T/A polymorphism within the RAGE gene was indicated to exhibit protective effects against cardiac death, angina and myocardial infarction in diabetic patients. The association between the $82 \mathrm{G} / \mathrm{S}$ polymorphism and ischemic stroke was studied by Cui et al (18), and their data suggested that in the subjects with the $82 \mathrm{~S} / \mathrm{S}$ genotype, the risk of ischemic stroke was significantly increased compared with the individuals with $82 \mathrm{G} / \mathrm{S}$ and $82 \mathrm{G} / \mathrm{G}$ genotypes (18). The results of a meta-analysis, consisting of 17 studies with 4,343 patients and 5,402 controls, which examined the $-429 \mathrm{~T} / \mathrm{C},-374 \mathrm{~T} / \mathrm{A}$ and G82S SNPs in the RAGE gene, were consistent with the results of the present study, which demonstrated that there were no statistically significant associations between polymorphisms and cardiovascular diseases (42). In a similar study examining the association between coronary artery disease and -374T/A and G82S RAGE polymorphisms in Turkish subjects, no significant difference was identified between patients and controls (43). Likewise, no significant difference was observed in the allele distributions of all 3 polymorphic regions between patients and control group in the present study.

In conclusion, the results of the present study indicated that there was no significant differences in $R A G E$ polymorphisms or allele distribution between patients with CSX and controls in the terms of CSX pathogenesis. To the best of our knowledge, this is the first study that aimed to examine and evaluate polymorphisms of the $R A G E$ gene in a Turkish CSX population. Conversely, there were certain limitations to the study: As CSX is a rare disorder, the number of patients participating in the study was limited. The study could also be developed further to evaluate the association between RAGE and several interacting parameters like AGE, S100/calgranulines and high-mobility group box-1 proteins by ELISA, and their roles in CSX. In addition, other polymorphic loci in the RAGE gene could be genotyped to determine the effect of those polymorphisms in CSX. Therefore, in order to comprehensively determine the contribution of $R A G E$ gene polymorphisms in CSX pathogenesis, additional studies with more participants are required, and the RAGE levels in patients with CSX may also be quantified.

\section{Acknowledgements}

Not applicable.

\section{Funding}

The present study was supported by the Scientific Research Projects of Istanbul University (project no. 3755).

\section{Availability of data and materials}

All data generated and analyzed during this study are included in this published article.

\section{Authors' contributions}

AGA, SÖ and BÖ designed the study. BD and BÖ designed and performed data collection. BÖ and DÖ performed the experiments. BO, BD and DÖ wrote the manuscript, and AGA and SÖ were responsible for the final edits of the manuscript.

\section{Ethics approval and consent to participate}

The study was approved by Ethics Committee of Cerrahpaşa Medical Faculty (approval no. 2015/279734) and was performed in accordance with Helsinki Declaration of World Health Organization. All the individuals who participated in the study provided written informed consent.

\section{Patient consent for publication}

All the individuals who participated in the study provided written informed consent for the publication of any associated data. 


\section{Competing interests}

The authors declare that they have no competing interests.

\section{References}

1. Singh M, Singh S, Arora R and Khosla S: Cardiac syndrome X: Current concepts. Int J Cardiol 142: 113-119, 2010

2. Vermeltfoort IA, Raijmakers PG, Riphagen II, Odekerken DA, Kuijper AF, Zwijnenburg A and Teule GJ: Definitions and incidence of cardiac syndrome X: Review and analysis of clinical data. Clin Res Cardiol 99: 475-481, 2010.

3. Rasmi Y, Raeisi S and Seyyed Mohammadzad MH: Association of inflammation and cytotoxin-associated gene a positive strains of helicobacter pylori in cardiac syndrome X. Helicobacter 17 116-120, 2012.

4. Agrawal S, Mehta PK and Bairey Merz CN: Cardiac syndrome X: Update 2014. Cardiol Clin 32: 463-478, 2014

5. Zuchi C, Tritto I and Ambrosio G: Angina pectoris in women: Focus on microvascular disease. Int J Cardiol 163: 132-140, 2013.

6. Laksanakorn W, Laprattanagul T, Wei J, Shufelt C, Minissian M, Mehta PK and Merz CNB: Cardiac rehabilitation for cardiac syndrome $\mathrm{X}$ and microvascular angina: A case report. Int J Case Rep Images 6: 239-244, 2015.

7. Lanza GA and Crea F: Primary coronary microvascular dysfunction: Clinical presentation, pathophysiology, and management Circulation 121: 2317-2325, 2010.

8. Lanza GA, Sestito A, Cammarota G, Grillo RL, Vecile E, Cianci R, Speziale D, Dobrina A, Maseri A and Crea F: Assessment of systemic inflammation and infective pathogen burden in patients with cardiac syndrome X. Am J Cardiol 94 40-44, 2004.

9. Cosín-Sales J, Pizzi C, Brown S and Kaski JC: C-reactive protein, clinical presentation, and ischemic activity in patients with chest pain and normal coronary angiograms. J Am Coll Cardiol 41: $1468-1474,2003$.

10. Dollard J, Kearney P, Clarke G, Moloney G, Cryan JF and Dinan TG: A prospective study of C-reactive protein as a state marker in Cardiac Syndrome X. Brain Behav Immun 43: 27-32, 2015.

11. Yang S, Wang H, Yang Y, Wang W, Jiang J, Zhao X, Du Q, Wang X, Yao Y, Shen H, et al: Association study of AGER gene polymorphism and hypertension in Han Chinese population. Gene 498: 311-316, 2012.

12. Kamide T, Kitao Y, Takeichi T, Okada A, Mohri H, Schmidt AM, Kawano T, Munesue S, Yamamoto Y, Yamamoto H, et al: RAGE mediates vascular injury and inflammation after global cerebral ischemia. Neurochem Int 60: 220-228, 2012

13. Kawai T, Kamide K, Ito N, Onishi M, Oguro R, Takeya Y, Tatara Y, Maekawa Y, Katsuya T, Ohishi M and Rakugi H: -374 T/A polymorphism in RAGE gene is associated with onset of diabetes mellitus, atherosclerosis, and renal dysfunction in patients with hypertension. Clin Exp Hypertens 35: 236-241, 2013.

14. Bucciarelli LG, Kaneko M, Ananthakrishnan R, Harja E, Lee LK, Hwang YC, Lerner S, Bakr S, Li Q, Lu Y, et al: Receptor for advanced-glycation end products: Key modulator of myocardial ischemic injury. Circulation 113: 1226-1234, 2006.

15. Kalousová M, Brabcová I, Germanová A, Jáchymová M, Matl I, Mestek O, Bandúr S, Zima T and Viklický O: RAGE polymorphisms, renal function and histological finding at 12 months after renal transplantation. Clin Biochem 42: 347-352, 2009.

16. Ng ZX, Kuppusamy UR, Iqbal T and Chua KH: Receptor for advanced glycation end-product (RAGE) gene polymorphism $2245 \mathrm{G} / \mathrm{A}$ is associated with pro-inflammatory, oxidative-glycation markers and sRAGE in diabetic retinopathy. Gene 521: $227-233,2013$

17. Liu L and Qiu XB: Association between the receptor for advanced glycation end products gene polymorphisms and coronary artery disease. Mol Biol Rep 40: 6097-6105, 2013.

18. Cui X, Chen H, Hou X, Wang S, Jayaram S and Zheng Z: Polymorphism of the RAGE affects the serum inflammatory levels and risk of ischemic stroke in a Chinese population. Cell Physiol Biochem 32: 986-996, 2013.

19. Falcone C, Campo I, Emanuele E, Buzzi MP, Zorzetto M, Sbarsi I and Cuccia M: Relationship between the -374T/A RAGE gene polymorphism and angiographic coronary artery disease. Int $\mathbf{J}$ Mol Med 14: 1061-1064, 2004.
20. Gao J, Shao Y, Lai W, Ren H and Xu D: Association of polymorphisms in the RAGE gene with serum CRP levels and coronary artery disease in the Chinese Han population. J Hum Genet 55: 668-675, 2010

21. Tripathi AK, Chawla D, Bansal S, Banerjee BD, Madhu SV and Kalra OP: Association of RAGE gene polymorphism with vascular complications in Indian type 2 diabetes mellitus patients. Diabetes Res Clin Pract 103: 474-481, 2014

22. Fox K, Garcia MA, Ardissino D, Buszman P, Camici PG, Crea F, Daly C, De Backer G, Hjemdahl P, Lopez-Sendon J, et al: Guidelines on the management of stable angina pectoris: Executive summary: The Task Force on the Management of Stable Angina Pectoris of the European Society of Cardiology. Eur Heart J 27: 1341-1381, 2006.

23. D'Agostino RB Sr, Vasan RS, Pencina MJ, Wolf PA, Cobain M, Massaro JM and Kannel WB: General cardiovascular risk profile for use in primary care: The Framingham Heart Study. Circulation 117: 743-753, 2008

24. Bruce RA: Exercise testing of patients with coronary heart disease. Principles and normal standards for evaluation. Ann Clin Res 3: 323-332, 1971.

25. Livak KJ and Schmittgen TD: Analysis of relative gene expression data using real-time quantitative PCR and the 2(-Delta Delta C(T)) method. Methods 25: 402-408, 2001.

26. Tousoulis D, Davies GJ, Asimakopoulos G, Homaei H, Zouridakis E, Ahmed N and Kaski JC: Vascular cell adhesion molecule-1 and intercellular adhesion molecule-1 serum level in patients with chest pain and normal coronary arteries (syndrome X). Clin Cardiol 24: 301-304, 2001.

27. Asbury EA and Collins P: Cardiac syndrome X. Int J Clin Pract 59: 1063-1069, 2005.

28. Arroyo-Espliguero R, Mollichelli N, Avanzas P, Zouridakis E, Newey VR, Nassiri DK and Kaski JC: Chronic inflammation and increased arterial stiffness in patients with cardiac syndrome X. Eur Heart J 24: 2006-2011, 2003.

29. Arroyo-Espliguero R and Kaski JC: Microvascular dysfunction in cardiac syndrome $\mathrm{X}$ : The role of inflammation. CMAJ 174: $1833,2006$.

30. O'Donnell CJ and Nabel EG: Genomics of cardiovascular disease. N Engl J Med 365: 2098-2109, 2011.

31. De Rosa S, Arcidiacono B, Chiefari E, Brunetti A, Indolfi C and Foti DP: Type 2 diabetes mellitus and cardiovascular disease: Genetic and epigenetic links. Front Endocrinol (Lausanne) 9:2, 2018.

32. Low Wang CC, Hess CN, Hiatt WR and Goldfine AB: Clinical update: Cardiovascular disease in diabetes mellitus: Atherosclerotic cardiovascular disease and heart failure in type 2 diabetes mellitus-mechanisms, management, and clinical considerations. Circulation 133: 2459-2502, 2016.

33. Williams SB, Cusco JA, Roddy MA, Johnstone MT and Creager MA: Impaired nitric oxide-mediated vasodilation in patients with non-insulin-dependent diabetes mellitus. J Am Coll Cardiol 27: 567-574, 1996.

34. Demir B, Önal B, Özyazgan S, Kandaz C, Uzun H, Açıksarı G, Uygun T, Opan S, Karakaya O and Akkan AG: Does inflammation have a role in the pathogenesis of cardiac syndrome X? A genetic-based clinical study with assessment of multiple cytokine levels. Angiology 67: 355-363, 2016.

35. Yan SF, Ramasamy R and Schmidt AM: Receptor for AGE (RAGE) and its ligands-cast into leading roles in diabetes and the inflammatory response. J Mol Med (Berl) 87: 235-247, 2009.

36. Li K, Zhao B, Dai D, Yao S, Liang W, Yao L and Yang Z: A functional p.82G $>\mathrm{S}$ polymorphism in the RAGE gene is associated with multiple sclerosis in the Chinese population. Mult Scler 17: 914-921, 2011.

37. Kucukhuseyin O, Yilmaz-Aydogan H, Isbir CS and Isbir T: Is there any association between GLY82 ser polymorphism of rage gene and Turkish diabetic and non diabetic patients with coronary artery disease? Mol Biol Rep 39: 4423-4428, 2012.

38. Li Y, Yang C, Ma G, Gu X, Chen M, Chen Y, Zhao B, Cui L and $\mathrm{Li} \mathrm{K}$ : Association of polymorphisms of the receptor for advanced glycation end products gene with COPD in the Chinese population. DNA Cell Biol 33: 251-258, 2014.

39. Cohen CR, Diel VB, La Porta VL, Rohde LE, Biolo A, Clausell N and Dos Santos KG: Association study of polymorphisms in the receptor for advanced glycation end-products (RAGE) gene with susceptibility and prognosis of heart failure. Gene 510: 7-13, 2012. 
40. Hooper J: Nurse education initiatives-making Project 2000 a reality. Managing the dynamic organisation. Nurse Educ Today 10: 380-384, 1990.

41. Falcone C, Geroldi D, Buzzi MP, Emanuele E, Yilmaz Y, Fontana JM, Vignali L, Boiocchi C, Sbarsi I and Cuccia M: The -374T/A RAGE polymorphism protects against future cardiac events in nondiabetic patients with coronary artery disease. Arch Med Res 39: 320-325, 2008.
42. Wang J, Zou L, Song Z, Lang X, Huang S, Lu F, Han L and Xu Z: Meta-analysis of RAGE gene polymorphism and coronary heart disease risk. PLoS One 7: e50790, 2012.

43. Aydoğan HY, Küçükhüseyin O, Tekeli A and Isbir T: Associations of receptor for advanced glycation end products -374T/A and Gly82 Ser and peroxisome proliferator-activated receptor gamma Pro12Ala polymorphisms in Turkish coronary artery disease patients. Genet Test Mol Biomarkers 16: 134-137, 2012. 